\title{
Seriality in Transmedia Storytelling: A Case Study of Halo
}

\begin{abstract}
This article posits that the concept of seriality is central to understanding and examining how transmedia projects balance between the need for the core medium to be understandable on its own while also making sure that transmedia extensions enhance the audience's narrative experience. First, in order to establish the main features of serial storytelling the paper contemplates seriality in contemporary television series, drawing extensively on the work of television theorist Jason Mittell. Second, these features are explored within the context of transmedia storytelling with a focus on how seriality across media affects the process of narrative comprehension. What then follows is a close reading of texts in the Halo transmedia franchise from a cognitive perspective and its transposition to narrative. The analysis shows that serialization across media, especially event serialization, creates narrative gaps in the Halo video games, the core texts of the franchise. To help the more casual fans to fill in these narrative gaps, the games feature internal redundancy in the form of diegetic retelling that transforms narrative enigmas into narrative statements.
\end{abstract}

Keywords: Transmedia Storytelling, Seriality, Narrative Comprehension, Video Game, Halo.

\section{Introduction}

Transmedia storytelling, the phenomenon of a shared fictional storyworld being expanded via different media, is by now familiar to most media consumers even if they are not aware of the term itself. The most widely known current example might be the Marvel Cinematic Universe, a media franchise that includes interrelated movies, television series as well as some comic books with each text expanding a shared storyworld. The term "transmedia storytelling" was coined and popularized by media theorist Henry Jenkins to describe a

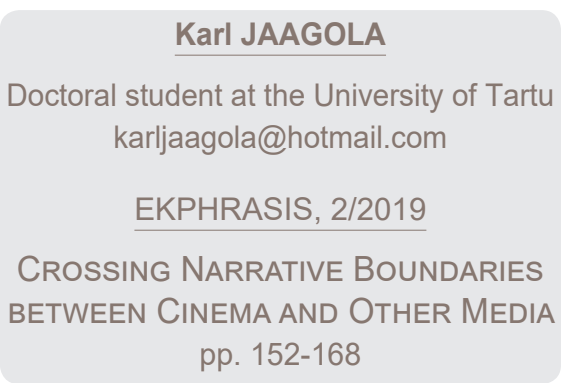

DOI:10.24193/ekphrasis.22.9

Published First Online: 2019/11/27 
new direction in transmedia production ("Transmedia Storytelling", n.pag.). While transmedia entertainment franchises had existed for decades, with entertainment companies using different media platforms to spread their intellectual properties, this mostly meant merchandising and producing adaptations of pre-existing stories in other media. Transmedia storytelling, on the other hand, aims to tell only new stories that remain consistent with all prior ones set in that particular shared imaginary world.

Much of the early writing on transmedia storytelling has concerned itself with the production side, i.e. how producers can best make use of this storytelling technique, and notably less consideration has been afforded to the issue of how audiences comprehend transmedia stories. This lack of attention seems especially noteworthy when we consider that one of the key questions for transmedia producers has been how to create texts that serve to provide the more hardcore fans, who consume texts across media, with integral story content while at the same time making texts in each medium understandable in their own right (Jenkins, "Transmedia Storytelling 101", n.pag.).

The lack of in-depth analyses of existing franchises is another major issue, which has been acknowledged by narratologist Marie-Laure Ryan, who says that in the field of narratology much of her writing as well as others' has been largely theoretical ("Transmedia Narratology" 7). Understandably, one of the reasons for this is that transmedia storytelling is still a relatively recent practice and to establish it as a concept on its own, many authors placed emphasis on its defining features and typology. Another reason for the lack of in-depth case studies is the large number of texts that each franchise can include (Ryan, "Transmedia Narratology" 7). This is why many of the existing case studies remain purely enumerative (Ryan, "Transmedia Narratology" 7).

With this in mind, the aim of this paper is to conduct a case study of the Halo transmedia franchise in order to make a contribution to the field of transmedia studies. The article explores how seriality works in the confines of transmedia projects and how it affects narrative comprehension.

\section{Seriality and Narrative Complexity}

In the most basic sense, seriality involves "the segmentation of a narrative into instalments that are released sequentially with, usually, a time lapse between the release of one instalment and the next" (Jones 527). In his 2015 book Complex TV: The Poetics of Contemporary Television Storytelling, Jason Mittell considers narrative complexity to be a mode of storytelling that emerged in the 90s "as an alternative to the conventional episodic and serial forms that have typified most American television since its inception" (17). To explain the differences between these three modes, Mittell breaks seriality down into four components: events, storyworld, characters and temporality (Complex TV 22). When we talk about serial storytelling, we usually refer "to the ongoing accumulation of events," which means that "what occurs in one episode will have happened to the characters and the storyworld as 
portrayed in future episodes" (Mittell, Complex TV 22-23). We are dealing with serial storytelling if characters remember and discuss events that happened in prior instalments and if those events inform the characters' actions and motivations and "have a persistent impact on the storyworld" (Mittell, "Operational Seriality" 231). Depending on whether events are serialized or not, we can distinguish between episodic and serialized form.

Conventional episodic form is typified by plot closure within every episode (Mittell, Complex TV 18). Additionally, the events of an episode do not have a significant impact on the stories of future instalments (Mittell, "Operational Seriality" 231). It should be noted, however, that even episodic series are usually serialized in the sense that they feature a serialized storyworld and characters (Mittell, Complex TV 22). This means that there is a consistent set of characters that persists from episode to episode and the setting (e.g. a fictionalized city) remains recognizably the same throughout the series, i.e. the storyworld is persistent (Mittell, Complex TV 22).

Conventional serial form, on the other hand, is characterized by a "continuing narrative that is not concluded until the end of the series" (Jones 527). Long-running serials like television soap operas delay resolution and closure endlessly with episodes "lacking a distinctive identity," each one instead serving as "just one step in a long narrative journey" (Mittell, "Narrative Complexity" 32). Every episode also ends with a suspense-inducing cliffhanger, "a moment of uncertainty" that motivates the audience to return for the next instalment (Mittell, Complex TV 237).

Keeping all of this in mind, narrative complexity in television can be understood as "an interplay between the demands of episodic and serial storytelling" (Mittell, Complex TV 19). For example, many episodic procedural television series, where each episode revolves around a crime or a medical case, also feature ongoing relationship stories that carry over from episode to episode (Mittell, "Narrative Complexity" 32). A series may also, for instance, alternate between standalone episodes as well as episodes that deal with an ongoing plot of some sort as is the case with the horror/ sci-fi TV series The X-Files (Mittell, Complex TV 19). Furthermore, a series may feature episodes with self-contained plots that at the same time still manage to advance an ongoing plotline that spans the entire season or series (Mittell, Complex TV 19).

Mittell distinguishes between major events, "which are central to the cause-andeffect chain of a plot", i.e. events that affect the characters and change the status quo of the storyworld and minor events that are "inessential to the plot" (Complex TV 23). The former are serialized while the latter are not. However, it is not always immediately clear under which category a particular event falls. For example, an even, which may initially appear as a minor one, meant to be forgotten, can have big ramifications on the overarching ongoing plot many instalments later (Mittell, Complex TV 24).

With regards to major events we can further distinguish between narrative statements, which raise questions only about the future, and narrative enigmas that raise questions "as to what precisely happened" in the past (Mittell, Complex TV 24). 
Narrative statements keep audiences hypothesizing about what will happen next while narrative enigmas drive us to hypothesize to fill in gaps in the story (Mittell, Complex TV 171).

\section{Seriality in Transmedia Storytelling}

Seriality as a concept has been touched on by Jenkins as well as a number of other transmedia scholars before. Jenkins has suggested that a transmedia storytelling project could take the form of "a hyperbolic version of the serial" where the story has "been dispersed not simply across multiple segments within the same medium, but rather across multiple media systems" ("Origami Unicorn", n.pag.). Ryan, on the other hand, rather firmly argues that transmedia storytelling "is not a serial" because instead of telling a single story the aim is to tell "a variety of autonomous stories, or episodes" ("Transmedia Storytelling" 4). In Ryan's opinion it would be annoying to have to read the beginning of a story in a novel, then get the middle part in a movie and finally play a video game to experience the ending ("Transmedia Storytelling" 4). Notably, many scholars, including Jenkins, agree that most of the time transmedia projects are indeed based on fictional worlds that are able to "sustain multiple interrelated characters and their stories" rather than telling one long continuous story ("Transmedia Storytelling 101" n.pag.). Christy Dena has suggested that we might then distinguish between transmedia projects that are made up of mono-medium stories and projects that exist as a "collection of media that tells one story" where audiences have to shift their attention between entries belonging to different media in order to properly comprehend the full story (3).

It seems that all three scholars are here thinking of transmedia storytelling in binary terms (i.e. conventional serial form or conventional episodic form) but because transmedia storytelling projects aim to tell stories that are standalone and at the same time integral parts of the overall narrative experience, then I propose that transmedia storytelling is best understood in terms of narrative complexity.

At this point one needs to explain what it means for a text to be an integral part of the overall narrative experience. Jenkins has borrowed the term "additive comprehension" from game designer Neil Young to refer to the way that "each new text adds a new piece of information which forces us to revise our understanding of the fiction as a whole" ("Transmedia Storytelling 101", n.pag.). For example, if after watching a film we pick up a novel that offers backstory information on certain characters from the movie, we might gain an entirely new perspective on the characters and their motivations in the film. For this to happen, however, the events experienced by the characters in the novel need to have a perceivable impact on those same characters in the movie. These events are either implicitly reflected in the characters' behavior and actions or they may be explicitly referenced by the characters. Alternatively, events from a prior franchise entry may not necessarily have an impact on a specific character but on the storyworld as a whole, which again 
in some way needs to be articulated in a future instalment. In other words, events have to be serialized across media to at least a certain degree for us to be able to talk about additive comprehension.

Explicit references to events that took place in previous franchise entries have the potential to create significant narrative gaps for the reader if they have not consumed those prior entries. The Matrix transmedia franchise is an example of this in that "the films present an image of the storyworld that is full of plot holes" that only entries in other media can fill (Ryan, "Transmedia Storytelling" 7). For instance, in the film The Matrix Reloaded a character named the Kid appears and it is through dialogue between him and Neo, the film series' main protagonist, that it is made clear that the two characters already know each other, but to find out how they met one would have to have watched one of the anime shorts that came out around the same time as the film (Ryan, "Transmedia Storytelling" 7).

The Matrix franchise is what we might call "balanced transmedia" in the sense that all the entries across media are integral parts of the overall narrative (Mittell, Complex TV 294). However, when we look at most existing transmedia franchises, especially ones that have grown from the more traditional media of film and TV, then we see that those two media are often privileged over others (Mittell, Complex TV 294). In the TV industry the central privileged text (a TV series) is called a "mothership". The mothership is privileged in the sense that texts in other media, which are often referred to as "transmedia extensions," are designed to be non-essential for understanding what is happening in the mothership (Mittell, Complex TV 294). This is done to not alienate the more casual members of the audience who only engage with the TV series, which usually generates the majority of the total revenue within a TVbased transmedia project (Mittell, Complex TV 295).

\section{Seriality's Effect on Narrative Comprehension}

From a cognitive perspective, we process a narrative by building a mental model of the storyworld and then continuing to update it as we are presented with new information (Ryan, "Narratology" 470). As Mittell explains it, "we learn about characters' backstories, relationships, interior motivations and beliefs" and "we gather information about the storyworld's geography, history, temporality and particular norms and rules" throughout a series (Complex TV 166). Furthermore, much of our comprehension process takes the form of drawing spatial, temporal and causal connections between these storyworld elements (Thon 46). This is mostly "preconscious and automatic" as we rely on cognitive schemata that we have developed "through accumulated experiences of consuming media, as well as norms of everyday perception and cognition" (Mittell, Complex TV 167).

As we engage with a narrative we constantly keep track of "what we know and what knowledge gaps might be filled" in the future (Mittell, Complex TV 167). In the case of a serialized TV series, for example, we may lack the necessary information to 
definitively fill in certain story gaps for years (Mittell, Complex TV 170). I would argue that with a mono-medium serialized story there is an expectation that major story gaps will be filled in eventually. In transmedia storytelling, however, being able to fill in those gaps is not guaranteed if we do not engage with all the media as The Matrix example demonstrates. Jenkins speculates that this is partly the reason as to why film critics, who had only engaged with the films and, therefore, missed out on essential bits of story information, were less favourable towards the sequel films at the time of their release (Convergence Culture 104). In other words, in the Matrix franchise events from the transmedia extensions were serialized to an extensive degree, which may have resulted in too big narrative gaps in the films.

Serialized TV series sometimes feature paratexts in the form of recap compilation episodes before the beginning of a new season "to refresh viewers' memories" and to help viewers who have missed episodes to fill in narrative gaps (Mittell, Complex TV 193). Alternatively, internal redundancy in the form of diegetic retelling, a popular technique in TV soap operas, can fulfil the same goal (Mittell, Complex TV 181). In essence, narrative information that has already been established earlier is repeated via naturalistic dialogue (Mittell, Complex TV 182). Theoretically, the same could be done in the mothership of a transmedia project to help the audience fill in narrative gaps even though they have not consumed the transmedia extensions.

\section{Methodology and the Halo Transmedia Franchise}

The previously mentioned financial realities of transmedia production, which result in the most lucrative medium serving as the mothership, have informed the choice of the franchise being analyzed in this paper. In TV- and film-based franchises, the mothership is likely to contain a minimal amount of references to events and characters from texts in other media, i.e. from the perspective of the mothership events and characters from other media are not serialized. This means that the results of analysis with regards to seriality between the mothership and transmedia extensions are likely to be predictable. Microsoft's Halo transmedia franchise, however, is based around a series of video games. Admittedly, the video game industry is currently the most profitable entertainment industry in the world, surpassing both TV and film, which means that in this instance the video games serve as the clear mothership. However, video games are a considerably younger storytelling medium.

First, as audiovisual media, film and TV have historically been far more robust storytelling media than video games. One of the main reasons for this is that up until relatively recently video games were in many ways limited by their underlying technology. During a panel titled "Building Transmedia Worlds in Halo 4," which was held at the 2012 Game Developers Conference, Armando Troisi, Narrative Director at 343 Industries, and Kevin Grace, Franchise Manager at 343 Industries, pointed out that while games are getting increasingly better at producing realistic human faces and facial animations, live-action content with real people still has a slight advantage 
when it comes to depicting human emotion (00:41:44-00:42:04). That is not to say that the level of graphical detail and animaton quality a game has determines whether we can consider it an apt vehicle for engaging storytelling, however. Many older games with lower graphical fidelity, at least by current standards, such as Bioware's Star Wars: Knights of the Old Republic (2003) are still considered to be exemplary pieces of storytelling.

Second, in addition to a narrative component, video games, by definition, also include a ludic component. Most games, with certain genres standing as exceptions, aim to offer players a challenge in the form of specific gameplay mechanics, rules and objectives. Despite an increasing emphasis on storytelling, games are still categorized into genres based on similar gameplay characteristics. Grace and Troisi note that in terms of gameplay the Halo games are first-person shooters "with lots of things blowing up" and, therefore, may not necessarily be the first choice for telling love stories or exploring the world's politics and backstories (00:44:10-00:44:41). In other words, gameplay can, to a certain extent, dictate and limit the type of stories that can be told.

When we consider these two factors, then we could theoretically expect extensions in a video game-based franchise to play a more prominent role in terms of providing relevant narrative content. Additionally, because the emphasis in the games may not strictly be on the narrative, they are allowed to include more story gaps than would usually be permissible in a TV- or film-based mothership.

From 2001 to 2010, the video games in Microsoft's franchise were developed by game studio Bungie with Microsoft overseeing the development of the overall franchise. After Microsoft and Bungie split in 2007, the tech company retained the Halo IP and established an internal game studio, 343 Industries, to create all future Halo-related content (Bass, n.pag.). The Halo franchise was launched in 2001 with the novel Halo: The Fall of Reach and the first-person shooter science fiction video game Halo: Combat Evolved. Since then, 10 more games, 24 more novels, 2 short story collections, 11 comic books, 2 live-action series, a collection of anime shorts and a serial podcast have been added to the franchise.

Through the process of close reading informed by a cognitive understanding of narrative the case study aims to find out whether transmedia extensions are serialized from the point of view of the mothership; to what degree they are serialized and what effect that serialization has on the process of narrative comprehension. Due to the spatial limitations of this article only the mainline games and select novels are covered as these are the two most prominent media in the franchise. The study does not concern itself with empirical players/readers but rather with a hypothetical generalized receiver, who relies on universal cognitive mechanisms to comprehend a narrative. 


\section{Analysis of Seriality in the Halo Transmedia Franchise}

From 2001 to 2007, the franchise alternated between novel and game releases. In 2001, the novel Halo: The Fall of Reach was published and was followed by the game Halo: Combat Evolved only two weeks later. 2003 saw the publication of the novel Halo: First Strike with the game Halo 2 following in 2004; and the third novel Halo: Ghosts of Onyx came out in 2006 with Halo 3 being released in 2007. In 2006, Marvel Comics also published a Halo graphic novel featuring four short stories set in the Halo universe. With the exception of the graphic novel, the rest of the six franchise entries were released in a chronological order.

The original game, Halo: Combat Evolved, starts in medias res with a human spaceship that is being chased by an alien force, known as the Covenant, unexpectedly arriving at a mysterious artificial ringworld, the titular Halo. Through a series of cutscenes, we are then introduced to Master Chief, a hulking green-armoured soldier and Cortana, his AI companion, who along with the rest of the crew need to board lifeboats and make an emergency landing on the Halo to escape the boarding parties of the Covenant. Through the course of Master Chief's and Cortana's adventures on the ringworld, the player learns that Halo is a weapon of some sort built by an ancient now-extinct race known as the Forerunners. It is also revealed that Halo holds a deep religious significance for the Covenant, who want to activate it. As the story progresses, Covenant warriors accidentally release a virulent species of parasitic organisms known as the Flood from containment chambers deep within the ring. Master Chief and Cortana then learn that Halo is just one among many, and when activated, the Halos destroy all sentient life in the galaxy to thwart the Flood infection from spreading. The game ends with Master Chief and Cortana blowing up the ringworld to make sure the weapon is not activated and that the Flood does not escape. In the final cutscene, both Chief and Cortana are out in space aboard a small starfighter overlooking the drifting ruins of Halo and one last exchange takes place between the two before credits roll:

Cortana: "We did what we had to do ... for Earth. An entire Covenant armada obliterated, and the Flood... we had no choice. Halo ... It's finished."

Master Chief': "No... I think we're just getting started."

(Halo: Combat Evolved)

Even though the game drops the player in the middle of the action, it does not feature events designed to make the player hypothesize about what happened in the past. The characters, of which there are only a few named ones, do not really mention any past events with the exception that there has been a war going on between the humans and the Covenant, who want to destroy Earth. They also do not talk about any interpersonal relationships. Based on sarcastic yet friendly banter between Cortana and Chief, we can infer that they know each other and are perhaps friends but outside of that we really know nothing about them. We can infer that Master Chief 
is an especially competent warrior because of how other soldiers address him and because he is trusted with important tasks, but his past, beliefs and inner motivations remain obscure to us. Throughout the game Cortana delivers most of the dialogue which exclusively concerns exposition about Halo and mission objectives. While the previously mentioned exchange between the two characters hints that there might be more to the story, it also serves to provide a sense of resolution. The player could, of course, hypothesize about aspects of the fictional world and where the story could go next, but the narrative itself does not really pose those questions.

Somebody who reads the novel Halo: The Fall of Reach, before playing the game, which would be the sequential way of consuming the franchise entries, would have a vastly different perspective on the game's events, however. The story in the first novel begins 35 years prior to the events of Halo: Combat Evolved and takes the reader through various stages of Master Chief's life leading right up to the start of the game. In the novel it is revealed that Master Chief, whose real name we learn to be John, was kidnapped along with 74 other children from different human colonies by a genius scientist Dr. Catherine Halsey whose goal is to train and biologically augment them to become the most lethal fighting force imaginable. Many of the human colonies are openly rebelling against the Earth's government and these super soldiers, Spartans, are designed to subdue these uprisings in the hopes of averting an all-out intergalactic civil war.

Eight years into the Spartans' training, however, humanity encounters the Covenant and John and his fellow Spartans gain a new purpose. The story then picks up a short time before the events of Halo: Combat Evolved. As most of the human colonies have fallen with the exception of the planet Reach, the last line of defence between the Covenant and Earth, a plan is concocted by Dr. Halsey for John and the Spartans to steal a Covenant ship, infiltrate the Covenant and kidnap their leaders so that they can be used as bargaining chips to end the war before the Covenant reaches Earth. Right before this plan is about to be put into effect, however, the Covenant finds Reach and launches a massive attack. Even though John and Cortana manage to escape on a ship, Dr. Halsey and the rest of the Spartans are left behind on the planet. The novel ends with John and Cortana arriving at the Halo, which is exactly where the story then picks up in Halo: Combat Evolved.

The novel notably establishes concrete backgrounds for both John and Cortana and provides further characterization, introduces additional characters like Dr. Halsey and Blue Team, John's most trusted friends and fellow Spartans, and explains the hierarchical structure and the nature of the Covenant. It also ends with what could be considered a cliffhanger as none of the major storylines are resolved and the fate of the characters is left up in the air.

If we consider the novel and the game together, then we can say that the storyworld as well as some of the characters are serialized because they persist across the texts. Additionally, events from the novel are serialized as well because the status quo of 
the storyworld at the beginning of the game is the direct result of the chain of events experienced by the characters in the novel. Together, the novel and the game could be viewed as an example of the conventional serial form because by the end of the novel its storylines are not resolved and the game picks up on one of those storylines right where it left off. Furthermore, having read the novel, we now know that the game does not, in fact, provide resolution to the overarching story because we still do not know the fates of half the characters from the novel and the Prophets and much of the Covenant still pose a threat to the protagonists. What is unusual for serialized form, however, is the fact that the characters in the game do not reference any of the events that happened in the novel.

Mittell says that "most classically episodic programs [...] ignore previous events rather than explicitly [...] deny their existence" (Complex TV 23). In this sense, the two instalments together operate more like episodic instalments in a series. While the storyworld reflects the impact of the events of the novel, characters' dialogue and actions do not. As Mittell notes, "oftentimes, frustration with a serialized program stems from moments when viewers' memories are more acute than those of characters" and viewers "wonder why characters do not seem to remember what happened previously to them and behave accordingly" (Complex TV 23). This certainly applies in this particular situation because one would expect Chief and Cortana to express a desire to return to Reach to save Dr. Halsey and the rest of the Spartans or to get back to the mission of kidnapping the Prophets. Arguably, this issue for the reader/player is further exacerbated by the game characters' lack of reference to what happened right before the events of the game.

Before moving on to further instalments of the franchise, it should also be pointed out that while we are dealing with a serialized storyworld between the novel and the game, the novel presents the storyworld in much greater detail. In the game quite a lot about the fictional world is left intentionally vague to the point where a player could invent an entirely different backstory for the characters and the conflict they are thrown into and not a lot in the game itself would contradict this player-invented backstory.

When we consider all of the above, then there does seem to be an attempt on the part of the storytellers to not confuse those who only play the game by not including references to any of the events in the preceding novel. However, this means that for the game-only part of the audience, a lot about the storyworld remains mysterious and unknown while those players who have read the novel may be left frustrated by the fact that characters in the game do not appear to remember events they just experienced. On the other hand, if we consider the novel not as a first instalment in a series but instead as a transmedia extension that accompanies a mothership, then the novel clearly provides additive comprehension as it gives the player a far deeper understanding of the storyworld and re-contextualizes the game's story, i.e. it makes it clear that the overall story does not end with the game. 
The third franchise entry, the novel Halo: First Strike, immediately picks up on both previously unresolved storylines from the end of Halo: Combat Evolved as well as from the end of Halo: The Fall of Reach. Characters from both previous texts return and the events of both franchise entries are explicitly serialized through the characters' dialogue, motivations and actions. The story of the novel involves Master Chief and Cortana rescuing Halsey and the Spartans stranded on Reach and attempting to destroy a Covenant fleet that is about to launch an attack on Earth. The novel ends with Chief, Cortana and Blue Team returning to Earth to inform them that the Covenant has finally learned of Earth's location. Although by the end of the novel the overarching human-Covenant war storyline is not resolved, the novel does not end with a cliffhanger as none of the characters are in any imminent danger and many of the minor plotlines from the first novel are wrapped up.

Halo 2's story then picks up some time after the events of Halo: First Strike with Chief stationed on a space station in Earth's orbit. While Halo 2 does introduce certain story elements into its narrative that had been first introduced in the novels (for example, the Prophets), once again the events of the two prior novels are not referenced by Chief or Cortana and characters like Halsey and members of Blue Team do not appear. A player who has not read the novels would also probably not question how Chief and Cortana got to Earth as they were both aboard a starfighter at the end of Halo: Combat Evolved. This is a gap in the narrative that can be easily filled in by the player without having to refer to any outside material.

In addition to Master Chief, Halo 2 introduces a second playable protagonist in the form of the Arbiter, a member of a warrior race, the Sangheili, that make up the military leadership of the Covenant. When Master Chief assassinates one of the three Prophets, the other two consider this a failure on the part of the Sangheili and order them to be exterminated by the other member races of the Covenant. By crossing paths with Master Chief, the Arbiter also learns the truth about Halos. The Prophets have lead all of their followers to erroneously believe that, by activating the Halos, all faithful members of the Covenant will achieve transcendence and be able to join their gods, the Forerunners. The Prophets' betrayal of the Sangheili and the truth that Halos are just a weapon system designed to destroy all life in the galaxy lead to a Covenant civil war, where the Arbiter and the Sangheili ally themselves with Chief and the rest of humanity.

Halo 3 concludes the human-Covenant story with the destruction of the remaining two Prophets as well as the Flood after which a peace is declared between the Covenant and humankind. The ending of the third game sees the Sangheili, now under the leadership of the Arbiter, heading back to their home planet. Chief and Cortana, however, are presumed to have been killed in action. The final cutscene reveals both to still be alive but stuck on a half-destroyed ship adrift in space, unable to get back to Earth. Cortana believes that it might take years for someone to find them and promises to keep watch as Chief enters a cryogenic chamber to wait for rescue. 
While a third novel, Halo: Ghosts of Onyx, was released in-between Halo 2 and Halo 3, it acts as a parallel story-which focuses on Dr. Halsey and Blue Team- to the one presented in Halo 2 and Halo 3. It should again be noted that the events and the characters in the novel were not referenced in Halo 3. The story of the novel, however, builds on all previous franchise entries.

Between 2008 and 2009, two additional spin-off games were released along with multiple comic books and novels, but the same degree of separation between the mothership and the extensions remained. In 2010, Bungie released their last Halo game titled Halo: Reach, which retold the events of the novel Halo: The Fall of Reach from the perspective of an entirely new cast of characters. For those gamers who had not ventured outside the games Halo: Reach would have been their first introduction to the character of Dr. Halsey.

When asked about story discrepancies between Halo: Reach and Halo: The Fall of Reach, Marcus Lehto, the Creative Director of the game, responded by saying that in the case of contradictions, the games, which make up the "Bungie canon," supersede materials in other media (Frushtick n.pag.). This type of hierarchical canon system where the games are internally considered to be the most important franchise entries would certainly explain why the games lack references to the novels. However, the relationship between the two media in terms of serialization during the 343 Industries era is considerably different from the one described so far with Grace and Troisi stressing that "everything counts in the universe" and that there are no "lesser tracks" (00:07:47-00:08:00). At the same time, they also claim to follow the mantra of "[N]o homework required" which means that casual fans should still be able to make sense of what happens in the games without having to consume other materials (00:27:18-00:29:03).

Halo 4, which was released in 2012, begins with a prologue cutscene showing Dr. Halsey in handcuffs in an interrogation room with a man who is asking her questions about the Spartan program and Master Chief. Through dialogue and flashbacks we learn that Dr. Halsey kidnapped children and turned them into Spartans. Halsey claims that her project saved the human race. The man retorts that Halsey is bending history in her favor as the Spartans were originally created to crush human rebellion, not to fight the Covenant. Here we might first consider players who have played only the games (including Halo: Reach, which features Dr. Halsey). For them, this scene provides new information about the origins of the Spartan program. We can also think of those players that have both played and read all the franchise entries mentioned so far. To them, this scene serves as a reminder of prior events via diegetic retelling in the form of naturalistic dialogue. For both groups this scene acts as a narrative enigma because it makes them question how and why Dr. Halsey has been arrested since her last chronological appearance in Ghost of Onyx. However, we may also consider a third subset of players. In 2011, the novel Halo: Glasslands was published, detailing the reasons and the circumstances of Halsey's arrest after the events of Halo 
3 and Halo: Ghosts of Onyx. For that subset of players, this opening cutscene does not act as a narrative enigma but instead as a narrative statement, which causes them to only hypothesize what will happen to Dr. Halsey next because they already know what the characters in the cutscene are talking about and they know what has caused the situation.

The story proper of Halo 4 then picks up four years after the events of Halo 3 with Chief being awakened by Cortana because their ship is being scanned by some kind of entity. Master Chief and Cortana then quickly come up against Covenant forces, including Sangheili, boarding the ship. Chief expresses dismay over this turn of events, saying " $[\mathrm{I}]$ thought we had a truce with the Covenant" to which Cortana replies "[A] lot can happen in four years," This is yet another instance where, for some subsets of players, this moment acts as a narrative enigma, while for others this encounter simply reminds them of what has already been revealed about the political climate of the galaxy via Halo: Glasslands and its follow-up, Halo: The Thursday War, which was published in 2012, shortly before Halo 4's release. The two novels notably also introduce the new leader of the Covenant, the creation of a new Spartan program and a number of characters who are referenced and appear in Halo 4.

Early in the game's story it is also revealed that Cortana is essentially losing her sanity because AIs start to deteriorate after seven years and Cortana was created eight years before. This piece of information about AIs is also revealed in the first novel but was not relevant to the plot until now. We might, therefore, think of this revelation as an example of an event which initially appeared to be a minor one turning out to be a major event that is serialized eleven years later.

If we again consider these examples in terms of seriality, then the mothership does, in fact, serialize events from the novels as characters in the game clearly remember those events, which can also be seen to have an impact on the state of the storyworld. Additionally, more characters persist across the different media texts than used to be the norm with earlier instalments. The question that now becomes relevant is whether there is an attempt on the part of the producers to avoid confusing the audience that mostly engages only with the mothership.

As has been established, Halo 4 presents players who have not read the novels with a number of narrative enigmas, i.e. gaps in the story, namely Dr. Halsey's arrest and the Covenant having seemingly turned against humanity during the four years that Chief and Cortana were missing. The narrative gap regarding Dr. Halsey is filled in by the end of Halo 4, which features a Halsey-centric story separate from Chief's and Cortana's adventure. Through a series of cinematic cutscenes, more about Dr. Halsey's past is revealed, with the ethics of the Spartan program brought up as an important issue. However, the overall situation regarding the Covenant is not explained to the player until Halo 5: Guardians which features the return of the Arbiter. This means that for the games-only audience this narrative enigma would have originally been drawn out for another three years. 
Similar examples of redundancy to help players fill in gaps in the narrative can also be pointed out in the fifth mainline game, Halo 5: Guardians. The game is the first to feature Chief's surviving Spartan friends, the Blue Team. The story begins with Chief and three of his friends already working as a team. It is not immediately established who these characters are so game-only players may have questions about these characters' past and about the nature of their relationship to Chief, while those who have read the novels would wonder how Chief got reunited with the members of Blue Team because this event has thus far not been shown in any of the games or the novels.

Arguably, the questions of the first subset of players are more important because not having answers to those questions creates a considerably bigger narrative gap. Therefore, through diegetic retelling, other characters establish the relationship between Chief and Blue Team in the game via the following exchange:

Vale: "I understand the Master Chief disobeying orders to find Cortana. But why the rest of Blue Team? They didn't know her."

Locke: "The members of Blue Team grew up together in the Spartan II program. They've registered more operations than any other fireteam in the UNSC."

Buck: "They're family."

Locke: "Exactly." (Halo 5: Guardians)

The answer to the question of how exactly Chief and Blue Team reunited prior to the events of Halo 5: Guardians, however, can only be found in the comic book series Halo: Escalation, published during the interim between the games.

Furthermore, when we consider Halo 5: Guardians as the mothership and the novels that feature Blue Team as its transmedia extensions then in addition to filling in a narrative gap, the novels also provide additive comprehension in that they "deepen audience engagement," which is another function of extensions outlined by Jenkins ("Transmedia 202", n.pag.). Somebody who had been reading about Blue Team for years would immediately be more invested in the game's narrative because they are already emotionally invested in the characters.

The last thing that should be noted about the novels and the games released by 343 Industries is that even though there is far more serialization across media than there was in the Bungie-produced games, there are no cliffhanger endings that are then resolved in another medium as was the case with Halo: Combat Evolved and the first two novels. The 343 Industries games and novels are, therefore, more reminiscent of television series that embrace narrative complexity in the sense that episodes with standalone plots still manage to further a greater ongoing story. 


\section{Conclusion}

The analysis of the Halo franchise revealed that in addition to the storyworld and characters, events are serialized across media as well. However, the franchise exhibited more examples of seriality across media once 343 Industries assumed control of the franchise from developer Bungie. While some characters and the storyworld are serialized across media in the Bungie games, events from transmedia extensions are not explicitly, and sometimes not even implicitly, serialized from the perspective of the games, potentially leading to frustration on the part of those audience members who consume texts in both media. This lack of explicit event serialization across media can be seen as the franchise producers' attempt to not compromise players' ability to comprehend the ongoing story that runs through the trilogy of games if they have not consumed the transmedia extensions.

Under the oversight of 343 Industries, events that take place in the transmedia extensions are, however, explicitly serialized across media, which leads to the creation of narrative enigmas or gaps in the later games' stories. While the games help players who have not consumed the transmedia extensions to fill in the principal major gaps in the story with redundant narration, some minor narrative gaps nevertheless remain unfilled. In the case of the games Halo 4 and Halo 5: Guardians, consuming the transmedia extensions beforehand or afterwards transforms narrative enigmas in the games into narrative statements.

\section{Works Cited}

\section{Primary Sources:}

343 Industries. Halo 4. Microsoft Studios, 2012. . Halo 5: Guardians. Microsoft Studios, 2015.

Bungie. Halo: Combat Evolved. Microsoft Game Studios, 2001. . Halo 2. Microsoft Game Studios, 2004. Halo 3. Microsoft Game Studios, 2007. Halo: Reach. Microsoft Game Studios, 2010.

Nylund, Eric. Halo: Ghosts of Onyx. Tor Books, 2006. . Halo: First Strike. The Definitive Edition, Tor Books, 2010. Halo: The Fall of Reach. The Definitive Edition, Tor Books, 2010.

Schlerf, Christopher. Halo: Escalation. Dark Horse Comics, 2013-2015.

Traviss, Karen. Halo: Glasslands. Tor Books, 2011. . Halo: The Thursday War. Tor Books, 2012.

\section{Secondary Sources:}

Bass, Dina. “The Halo Effect of Microsoft's Halo." Bloomberg, 2010, www.bloomberg.com/ news/articles/2010-08-26/the-halo-effect-of-microsofts-halo. Accessed 14 July 2019.

Dena, Christy. "Do You Have a Big Stick?" QUT ePrints, 2011, eprints.qut.edu.au/77679/. Accessed 15 Aug. 2019. 
Frushtick, Russ. “Bungie on the Contradictions Between 'Halo: Reach' and 'Halo: The Fall of Reach' Novel." MTV, 2010, www.mtv.com/news/2461855/bungie-on-the-contradic tions-between-halo-reach-and-halo-the-fall-of-reach-novel/. Accessed 29 Oct. 2019.

Grace, Kevin and Armando Troisi. "Building Transmedia Worlds in Halo 4." GDC Vault, 2012, www.gdcvault.com/play/1016603/Building-Transmedia-Worlds-in-Halo. Accessed 22 July 2019.

Jenkins, Henry. "Transmedia Storytelling." MIT Technology Review, 2003. www. technologyreview.com/s/401760/transmedia-storytelling/. Accessed 27 Oct. 2019.

. Convergence Culture: Where Old and New Media Collide. New York University Press, 2006.

. "Transmedia Storytelling 101." Confessions of an Aca-Fan, 2007. henryjenkins. org/2007/03/. Accessed 11 July 2019.

. "Revenge of the Origami Unicorn: The Remaining Four Principles of Transmedia Storytelling." Confessions of an Aca-Fan, 2009. henryjenkins.org/blog/2009/12 /revenge_ of_the_origami_unicorn.html. Accessed 29 July 2019.

. "Transmedia 202: Further Reflections." Confessions of an Aca-Fan, 2011, unpaginated. henryjenkins.org/2011/08/defining_transmedia_further_re.html. Accessed 3 Aug. 2019.

Jones, Sara Gwenllian. "Serial form." The Routledge Encyclopedia of Narrative Theory. Edited by David Herman, Manfred Jahn and Marie-Laure Ryan, Routledge, 2010, p. 527.

Mittell, Jason. Complex TV: The Poetics of Contemporary Television Storytelling. New York University Press, 2015.

. "Narrative Complexity in Contemporary American Television." The Velvet Light Trap, no. 58, 2006, pp. 29-40.

. "Operational Seriality and the Operation of Seriality." The Edinburgh Companion to Contemporary Narrative Theories, edited by Zara Dinnen and Robyn Warhol, Edinburgh University Press, 2018, pp. 227-38.

Ryan, Marie-Laure. "Narratology and Cognitive Science: A Problematic Relation." Style, vol. 44, no. 4, 2010, pp. 469-95.

. "Transmedia Storytelling: Industry Buzzword or New Narrative Experience?" Storyworlds, vol. 7, no. 2, 2015, pp. 1-20, www.academia.edu/37058566/ Transmedia_ Storyworlds_Industry_Buzzword_or_New_Narrative_Experience, Accessed 25 July 2019.

. "Transmedia narratology and transmedia storytelling." Artnodes, no. 18, 2016, pp. 1-10, artnodes.uoc.edu/articles/abstract/10.7238/a.v0i18.3049/, Accessed 17 Aug. 2019.

Thon, Jan-Noël. Transmedial Narratology and Contemporary Media Culture. University of Nebraska Press, 2016. 


\section{Acknowledgement}

Research for this paper was supported by the Estonian Research Council (Grant 1481, "The Role of Imaginary Narrative Scenarios in Cultural Dynamics") and by the European Union Regional Development Fund (Center of Excellence in Estonian Studies).

Karl Jaagola is a doctoral student and a junior research fellow at the Faculty of Arts and Humanities of the University of Tartu. His main research fields are transmedia studies, cognitive narrative studies and game studies, and his current research involves the examination and re-evaluation of established principles of transmedia storytelling through the in-depth and comprehensive analysis of the video gamebased Halo transmedia franchise. Prior to this project Karl's research was focused on the mediality of contemporary video games and the ways in which they borrow filmic storytelling techniques while at the same time differing from films due to their non-unilinear narrative structures and game-specific conventions. 